\title{
Expression of STAT3 and vasculogenic mimicry in gallbladder carcinoma promotes invasion and metastasis
}

\author{
HONGBING ZHOU $^{1,2}$, YIN YUAN ${ }^{2}$ and HAIXIN QIAN ${ }^{1}$ \\ ${ }^{1}$ Department of General Surgery, The First Affiliated Hospital of Soochow University, Suzhou, \\ Jiangsu 215006; ${ }^{2}$ Department of Hepatobiliary Surgery, The Fifth Affiliated Hospital of \\ The Medical School of Nantong University, Taizhou, Jiangsu 225300, P.R. China
}

Received November 20, 2020; Accepted April 14, 2021

DOI: $10.3892 / \mathrm{etm} .2021 .10170$

\begin{abstract}
Surgical treatment of gallbladder carcinoma remains challenging, while targeted therapy has been demonstrated to have potential. In the present study, the effect of signal transducer and activator of transcription 3 (STAT3) expression and vasculogenic mimicry (VM) on the occurrence and development of gallbladder carcinoma was evaluated. A total of 72 patients with gallbladder carcinoma and 10 patients with chronic cholecystitis were examined. Immunohistochemical staining was performed to determine the positive expression rates of STAT3. Periodic acid Schiff CD34 double staining was performed to detect VM in the gallbladder carcinoma group. STAT3 expression and VM in gallbladder carcinoma tissues was compared among patients with different clinical characteristics. In postoperative patients with gallbladder cancer, the relationship of the postoperative recurrence time with STAT3 expression and VM was assessed. STAT3 expression in gallbladder carcinoma tissue was significantly higher than that in cholecystitis tissue $(\mathrm{P}<0.05)$. STAT3 expression levels and VM were positively correlated in gallbladder carcinoma tissue. STAT3 protein expression in gallbladder carcinoma tissues differed significantly among patients with different degrees of differentiation and clinical stages $(\mathrm{P}<0.05)$. Among the 51 patients who completed the 3-year follow-up, the mean time to relapse was 17.353 and 35.647 months in those with high and low STAT3 expression, respectively, with significant differences $(\mathrm{P}<0.05)$. The VM structure was detected in 47 cases $(92.15 \%)$ and not detected in four cases $(7.84 \%)$, which exhibited no immediate recurrence after surgery, and
\end{abstract}

Correspondence to: Dr Haixin Qian, Department of General Surgery, The First Affiliated Hospital of Soochow University, 1 Shizi Street, Suzhou, Jiangsu 215006, P.R. China

E-mail: 3410634395@qq.com

Abbreviations: STAT3, signal transducer and activator of transcription 3; VM, vasculogenic mimicry; EDV, endothelialdependent vessels; EMT, epithelial to mesenchymal transition

Key words: STAT3, vasculogenic mimicry, gallbladder carcinoma, tumor development, endothelial-dependent vessel the difference in the mean postoperative recurrence time was significant ( 22.38 vs. 36.00 months, respectively; $\mathrm{P}<0.05)$. In gallbladder carcinoma tissues, a lower degree of differentiation, higher malignancy degree and higher clinical stage were associated with higher expression of STAT3 and VM. Thus, STAT3 may promote VM formation in the process of tumor occurrence, development and metastasis. Therefore, STAT3 as a regulatory target, may inhibit the proliferation and invasion of tumor cells and block the development of VM, thereby representing a suitable target for antitumor angiogenesis therapy.

\section{Introduction}

One of the most common malignant tumor types of the biliary system is gallbladder carcinoma. Its major clinical manifestations include right upper abdominal pain, gallbladder enlargement, jaundice, nausea and vomiting, upper gastrointestinal bleeding, and cachexia signs such as low fever and wasting (1). With an annual incidence of $\sim 528$ per 100,000 individuals, China is a region with a high incidence of this cancer type (2). Surgical resection remains the preferred treatment for gallbladder carcinoma and postoperative adjuvant therapy has an important role in prolonging the survival time of patients. However, it is difficult to detect gallbladder carcinoma at an early stage because of its high malignancy and lack of specific symptoms. Diagnosis is typically associated with lymph node metastasis and liver metastasis. This leads to a low resection rate and poor prognosis, causing serious harm to human health (3). Signal transducer and activator of transcription 3 (STAT3) is one of the most important members of the STAT family and is the junction point of numerous carcinogenic signaling pathways. It has been well established that all successful tumors must undergo neovascularization (4). Vascular endothelial growth factor (VEGF) is one of the most important of all known inducers of angiogenesis. Previous evidence indicated that constitutive STAT3 activation directly promotes VEGF expression and stimulates tumor angiogenesis, and STAT3 is a direct factor in VEGF expression (5). Multiple studies have confirmed that STAT3 is continuously activated in numerous tumor cell types (6-8). It is closely related to the proliferation, invasion, immune escape and microvascularization of tumor cells (9-12). Upregulation of STAT3 expression may promote the early abnormal division of gallbladder 
epithelial cells by improving the activation degree of the tumor signaling pathway in tumor cells and transcriptional activation degree of tumor-associated genes (13).

Vasculogenic mimicry (VM), which is a vascular structure formed by tumor cells that mimics the function of endothelial cells, is a novel tumor vascular model (14). Angiogenesis, mosaic vessels and VM are considered as the three major factors promoting intracranial vascular formation in tumors. These factors are common in melanoma, inflammatory breast cancer, osteosarcoma, liver cancer and other highly invasive malignant tumor types. VM involves tumor cells that have been generated internally and gradually integrated with endothelial-dependent vessels (EDV) of host origin. The intermediate states of these two vessels are known as mosaic vessels, which are transitional vessels surrounded by tumor and host endothelial cells (15).

Increasing evidence has indicated that inflammatory signals in the diseased tissue environment are closely related to the occurrence, maintenance and development of tumors (16). Gallbladder carcinoma is frequently associated with gallstones. Stimulation of calculi and the concurrent inflammation of gallbladder mucosa may lead to the occurrence of gallbladder carcinoma. STAT3 is a key molecule mediating inflammation in tumors (17). Its status in the tumor microenvironment determines the immune response, including whether the tumor is promoted or inhibited. Its continued activation in tumor cells promotes cell proliferation and survival (18). Therefore, STAT3, as a control target, may inhibit the proliferation and invasiveness of tumor cells, similar to the effects of inhibiting the development of VM and EDV. STAT3 may be a useful target for antitumor angiogenesis therapy. Therefore, in the present study, the gallbladder tissue expression and correlation of STAT3 and VM were analyzed in patients with gallbladder carcinoma. The relationship between the occurrence and development of gallbladder carcinoma and its potential for targeted gene therapy were examined. Targeted gene therapy may help to improve treatment strategies and long-term curative effects in patients with gallbladder carcinoma.

\section{Materials and methods}

Patients, tissue samples and clinical data collection. The experimental study of clinical tissue samples was reviewed and approved by the Ethics Committee of the Fifth Affiliated Hospital of the Medical School of Nantong University (Taizhou, China). All patients participating in the study or their family members signed an informed consent form for the clinical study. Paraffin tissue samples were collected from 72 patients with gallbladder carcinoma and 10 cases of chronic cholecystitis at the Fifth Affiliated Hospital of the Medical School of Nantong University between January 2013 and December 2016. The study cohort for gallbladder carcinoma included 40 female patients and 32 male patients (male-to-female ratio, 1:1.4) with an age range of 42-82 years. The male-to-female ratio of patients with chronic cholecystitis was 1:1.5, with an age range of 45-76 years. Only subjects with complete medical records were enrolled. None of the patients included in the study had undergone any preoperative chemotherapy, radiation therapy or other treatment. A total of 72 wax blocks of gallbladder cancer and 10 wax blocks of chronic cholecystitis tissue were selected, fixed on a slicer and sliced to $4-\mu \mathrm{m}$ sections. The manual slicer was used with uniform rotation, the selected slice was brushed and immersed in water. After the sections were fully flattened on the surface of warm water at $45^{\circ} \mathrm{C}$, they were picked up with anti-slip slides, and then placed in an electrically heated constant temperature air drying oven at $65^{\circ} \mathrm{C}$ for 30-60 min until the sections were completely dried. All tissue samples were pathologically diagnosed. There were 18 cases of highly differentiated carcinoma, 25 cases of moderately differentiated carcinoma, and 29 cases of poorly differentiated carcinoma according to the TNM staging criteria published by the International Union Against Cancer (19). Follow-up of the patients was mainly performed via telephone and outpatient reexamination. The postoperative recurrence time was calculated by month, and the truncation time was from the first operation to the recurrence time. The cut-off date for follow-up was December 2019.

Main reagents. Rabbit anti-human STAT3 monoclonal antibody (1:100; cat. no. BSM-52235R; BIOSS); Diaminobenzidine (DAB) color reagent (Beijing Zhongshan Jinqiao Biotechnology Co., Ltd.); poly-l-lysine coated slides (cat. no. AR1065; Wuhan Boster Biological Technology, Ltd.); HRP-conjugated AffiniPure goat anti-rabbit IgG testing kit (cat. no. BA1054; Wuhan Boster Biological Technology, Ltd.); Periodic Acid-Schiff Stain kit, (cat. no. DG0005; Beijing Leigen Biotechnology Co., Ltd.); 5\% BSA (cat. no. AR0004; Wuhan Boster Biological Technology, Ltd.) and normal goat serum (cat. no. AR0009; Wuhan Boster Biological Technology, Ltd.)

Immunohistochemical staining. Gallbladder lesion tissues were taken from patients with gallbladder carcinoma and chronic cholecystitis. After baking, tissue specimens were dewaxed and hydrated, washed with PBS for $20 \mathrm{~min}$ at room temperature, incubated in EDTA antigen retrieval solution $(1 \mathrm{mM})$ at $95-100^{\circ} \mathrm{C}$ for $\sim 15 \mathrm{~min}$ and blocked with $5 \%$ BSA. Rabbit anti-human STAT3 monoclonal antibody was used as a primary antibody, incubated at $4{ }^{\circ} \mathrm{C}$ overnight, followed by washing with PBS. Subsequently, the sample was incubated with goat anti-rabbit antibody labeled with horseradish peroxidase (HRP) (diluted 1:1,000) at room temperature for $2 \mathrm{~h}$ and then washed with PBS three times for $5 \mathrm{~min}$. DAB solution was added, color development was checked under the microscope and the solution was washed with distilled water after satisfactory color development. After counterstaining for 1 min with hematoxylin, the slides were dehydrated in sequential ethanol gradients $(75,80$ and $100 \%)$ of 1 min each. The slides were incubated in xylene until transparent and they were sealed with neutral gum. As the negative control, normal goat serum was used instead of primary antibody, while the other steps remained unchanged, and the result was negative. As the blank control, PBS was used instead of primary antibody; the other steps remained unchanged, and the results were negative.

Periodic acid Schiff (PAS)/CD34 double staining. For double staining, CD34 (1:100; cat. no. ab8536; Abcam) staining was performed first and the specific labeling steps were the same as those described above. CD34 staining was completed by color development with DAB and subsequently, the PAS staining procedure was performed. For this, 1\% PAS was 
Table I. Age and sex distribution of patients in chronic cholecystitis and gallbladder carcinoma.

\begin{tabular}{lcc}
\hline Factor & Chronic cholecystitis & Gallbladder carcinoma \\
\hline Sex, n $(\%)$ & & \\
Male & $4(40 \%)$ & $30(41.7 \%)$ \\
Female & $6(60 \%)$ & $42(58.3 \%)$ \\
Male-to-female ratio & $1: 1.5$ & $1: 1.4$ \\
Age, years $(\%)$ & & $32(44.4)$ \\
$<60$ & $6(60 \%)$ & $40(55.6)$ \\
$\geq 60$ & $4(40)$ & 59.53 \\
Median age, years & 57.76 & 0.950 \\
\hline
\end{tabular}

The P-value for sex was calculated using Wilcoxon rank sum test. The P-value for age was calculated using independent-sample t-test.

oxidized under the exclusion of light for $10 \mathrm{~min}$. Slices were rinsed with double distilled water after running tap water. At room temperature, drops of PAS stain were added to the slices, followed by incubation in the dark for $20 \mathrm{~min}$. PAS solution was rinsed off thoroughly with running water. The nucleus was stained with hematoxylin at room temperature for $5 \mathrm{~min}$, and hematoxylin was rinsed off with running water. The sections underwent dehydration with an ethanol gradient (70, 80, 90, 95 and 100\%) and made transparent with xylene, followed by sealing with neutral gum.

VM is surrounded by tumor cells and its wall is rich in extracellular matrix (20). VM structures may be stained purplish red by PAS. CD34 immunostaining is negative due to lack of endothelial involvement. Therefore, in PAS/CD34 double staining, the lumen structure with PAS-positive and CD34-negative staining may be used to identify VM-positive structures with red blood cells in them.

Immunohistochemical staining result scoring. For each tissue slice, staining results were determined and the pathological diagnosis was made by two senior physicians independently, who were blinded to the clinicopathological data and research content. STAT3 protein is mainly located in the cytoplasm and was determined from 10 randomly selected views at high magnification (x100), counting 1,000 cells. The results were obtained using a semi-quantitative integral method (21), according to the immunohistochemical score for the intensity of each section and the proportion of positive cells. Color intensity was scored as follows: No color, 0 points; light yellow, 1 point; brownish yellow, 2 points with; Tan, 3 points. The percentage of stained cells was scored as follows: $<5 \%$, $0 ; 5-24 \%, 1 ; 25-49 \%, 2 ; 50-74 \%, 3 ; 75-100 \%, 4$. Multiplication of the two values was used to obtain the semi-quantitative results. A final score of 0-2 was defined as negative expression, while a score of $>2$ was considered to indicate positive expression.

Statistical analysis. SPSS version 21.0 statistical software (IBM Corp.) was used for data analysis and the results were expressed as the mean \pm standard deviation. Wilcoxon rank sum test and independent-sample Student's t-test were used to analyze differences in age and sex distribution between the two groups of patients. Pearson's $\chi^{2}$ test and Fisher's exact test were used to statistically analyze the expression levels of STAT3 in the tissues from the two groups of patients. Spearman rank correlation analysis method was also used to analyze the correlation between STAT3 and VM expression levels in gallbladder carcinoma tissues. The association between the two indicators and clinicopathological data was also statistically analyzed with Pearson's $\chi^{2}$ test and Fisher's exact test methods. The cut-off value for STAT3 was calculated using a receiver operating characteristic curve. Survival curves were plotted using Kaplan-Meier analysis and the log-rank test was used to compare differences between the survival curves. $\mathrm{P}<0.05$ was considered to indicate a statistically significant difference.

\section{Results}

Expression of STAT3 in gallbladder carcinoma and cholecystitis tissues. In the present study, STAT3 expression was assessed in 72 cases of primary gallbladder carcinoma and 10 cases of chronic cholecystitis using immunohistochemistry. The male-to-female ratio of patients with gallbladder carcinoma was 1:1.4, with an age range of $42-82$ years. The male-to-female ratio of patients with chronic cholecystitis was 1:1.5, with an age range of 45-76 years (Table I). The median age of the patients with gallbladder carcinoma was 59.53 years and the median age of the patients with chronic cholecystitis was 57.76 years (Table I). There was no significant difference in age $(\mathrm{F}=0.004, \mathrm{P}=0.950$; Table $\mathrm{I})$ or sex between the two groups (sum of ranks: 2,982 in the gallbladder carcinoma group and 421 in the chronic cholecystitis group; $\mathrm{P}=0.921$; Table I). The positive expression rate of STAT3 in gallbladder carcinoma and chronic cholecystitis was 83.3 and $10.0 \%$, respectively. Fisher's exact test indicated that the expression rate of STAT3 in gallbladder carcinoma tissues was significantly higher than that in chronic cholecystitis tissues $(\mathrm{P}<0.001$; Table II and Figs. 1 and 2).

Association between STAT3 expression in gallbladder carcinoma and clinicopathological factors. Clinical stage analysis indicated that the STAT3 expression rate in gallbladder carcinoma of stages I-III was $73.7 \%$ and that of stages IV/V was $94.1 \%$. This suggested that a higher clinical stage was significantly positively associated with STAT3 expression (Fisher's exact test $\mathrm{F}=5.867, \mathrm{P}=0.027$; Table III). The rate of 
Table II. Expression of STAT3 in gallbladder carcinoma and chronic cholecystitis tissues.

\begin{tabular}{lcccc}
\hline & & \multicolumn{2}{c}{ STAT3 expression } & \\
\cline { 3 - 5 } Group & $\mathrm{n}$ & Positive & Negative & Positive rate $(\%)$ \\
\hline Gallbladder carcinoma & 72 & 60 & 12 & 83.3 \\
Chronic cholecystitis & 10 & 1 & 9 & 10.0 \\
\hline
\end{tabular}

The P-value was calculated by Fisher's exact test. STAT3, signal transducer and activator of transcription 3.

A

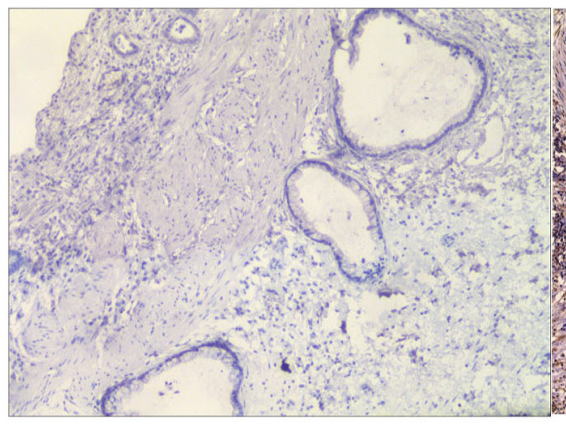

B

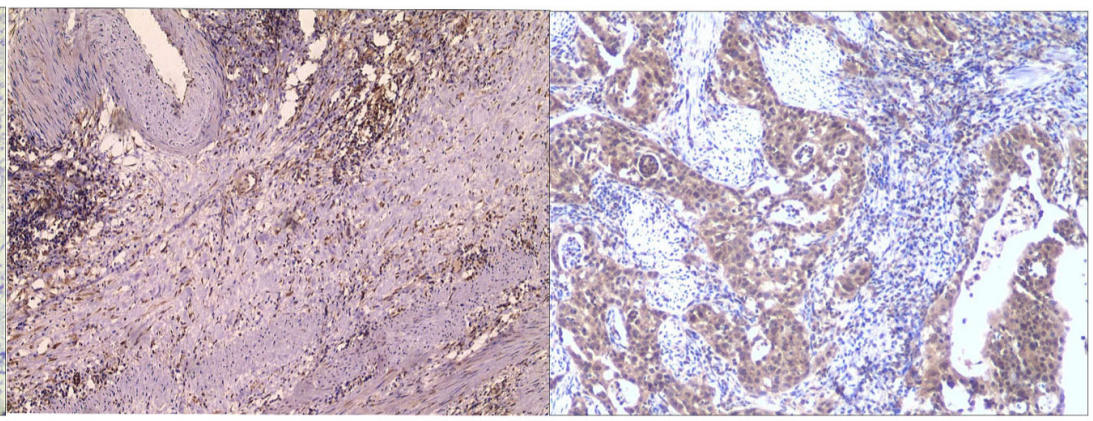

Figure 1. (A) Negative immunohistochemistry staining for STAT3 in gallbladder carcinoma; (B) low expression of STAT3 in gallbladder carcinoma, light brown-yellow stain; (C) high expression of STAT3 in gallbladder carcinoma, dark brown-yellow stain (magnification, x100). STAT3, signal transducer and activator of transcription 3 .

A

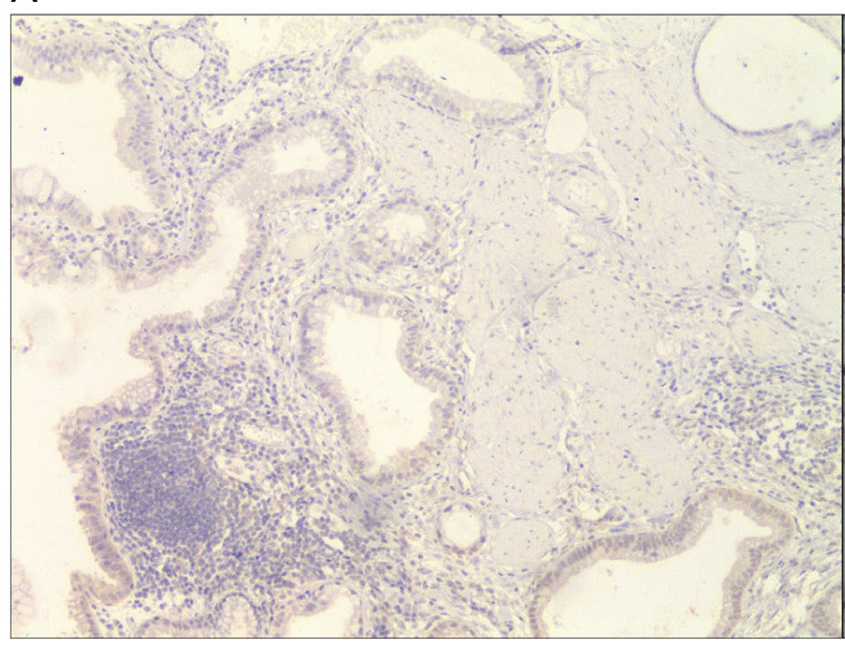

B

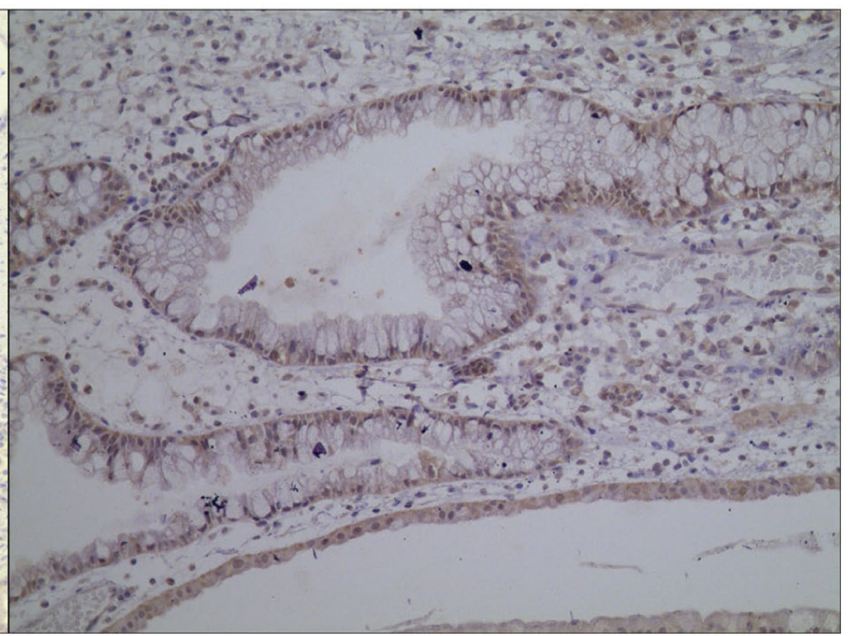

Figure 2. (A) Negative immunohistochemistry staining for STAT3 in chronic cholecystitis; (B) low expression of STAT3 in chronic cholecystitis (magnification, x100). STAT3, signal transducer and activator of transcription 3.

STAT3 expression in well, moderately and poorly differentiated gallbladder carcinoma tissues was 66.7, 80.0 and $96.6 \%$, respectively, and accordingly, the rate of STAT3 expression significantly increased with decreasing histological grade (Fisher's exact test $\mathrm{P}=0.017$; Table III). There was no significant difference in STAT3 expression in gallbladder carcinoma tissues between different subgroups according to sex, age and lymph node metastasis (sex, $\chi^{2}=0.411, P=0.521$; age, $\chi^{2}=0.180$, $\mathrm{P}=0.671$; lymph node metastasis, Fisher's exact test $\mathrm{F}=4.292$, $\mathrm{P}=0.061$; Table III).
Association between VM in gallbladder carcinoma and clinicopathological factors. In the present study, 58 cases of gallbladder carcinoma presented with typical VM structures as follows: i) Tumor cells connected with each other through a cell bridge to form the vascular lumen, in which red blood cells or free endothelial cells were occasionally observed; and ii) the tumor cells were stained using PAS into a purplish red net-like structure (Fig. 3). Statistical analysis revealed no significant difference in VM in gallbladder carcinoma tissues between different sexes, ages and lymph node metastasis 
Table III. Association between STAT3 and clinicopathological factors of patients with gallbladder carcinoma.

\begin{tabular}{|c|c|c|c|c|c|c|}
\hline \multirow[b]{2}{*}{ Factor } & \multirow[b]{2}{*}{$\mathrm{n}$} & \multicolumn{2}{|c|}{ STAT3 expression } & \multirow{2}{*}{$\begin{array}{l}\text { Positive } \\
\text { rate }(\%)\end{array}$} & \multirow{2}{*}{$\begin{array}{c}\chi^{2} / \text { Fisher's exact } \\
\text { test value }\end{array}$} & \multirow[b]{2}{*}{ P-value } \\
\hline & & Positive & Negative & & & \\
\hline Sex & & & & & 0.411 & 0.521 \\
\hline Male & 30 & 24 & 6 & 80.0 & & \\
\hline Female & 42 & 36 & 6 & 85.7 & & \\
\hline Age (years) & & & & & 0.180 & 0.671 \\
\hline$<60$ & 32 & 26 & 6 & 81.3 & & \\
\hline$\geq 60$ & 40 & 34 & 6 & 85.0 & & \\
\hline Histological grade & & & & & 7.645 & 0.017 \\
\hline Well & 18 & 12 & 6 & 66.7 & & \\
\hline Moderate & 25 & 20 & 5 & 80.0 & & \\
\hline Poor & 29 & 28 & 1 & 96.6 & & \\
\hline Lymph node metastasis & & & & & 4.292 & 0.061 \\
\hline $\mathrm{N} 1 / \mathrm{N} 2$ & 54 & 48 & 6 & 88.9 & & \\
\hline N0 & 18 & 12 & 6 & 66.7 & & \\
\hline Clinical stage & & & & & 5.867 & 0.027 \\
\hline I-III & 38 & 28 & 10 & 73.7 & & \\
\hline $\mathrm{IV} / \mathrm{V}$ & 34 & 32 & 2 & 94.1 & & \\
\hline
\end{tabular}

P-values for age and sex were calculated by Pearson's $\chi^{2}$ test and those for histological grade, lymph node metastasis and clinical stage were calculated by Fisher's exact test. STAT3, signal transducer and activator of transcription 3.

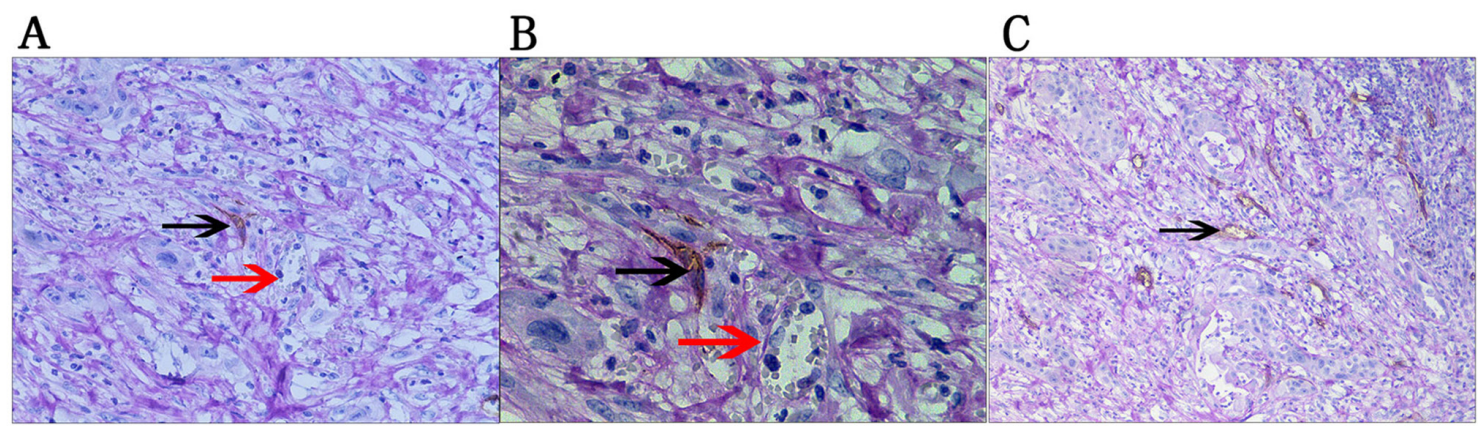

Figure 3. Representative images of positive immunohistochemistry staining for VM in gallbladder carcinoma. (A) magnification, x100; (B) magnification, x400 (black arrows indicate normal vascular endothelial cells with CD34-positive stain and brown-yellow color, and the lumen contains red blood cells; red arrows indicate the VM-positive structure and tumor cells surround the lumen; the periodic acid Schiff-positive area is rose-colored and CD34-negative, and red blood cells may be observed in the lumen). (C) Representative image of negative immunohistochemistry staining for VM in gallbladder carcinoma. Magnification, x100. The black arrows indicate normal vascular endothelial cells with CD34-positive stain and brown-yellow color, and the lumen contains red blood cells. VM, vasculogenic mimicry.

status $\left(\mathrm{sex}, \chi^{2}=0.010, \mathrm{P}=0.920 ;\right.$ age, $\chi^{2}=0.217, \mathrm{P}=0.641$ lymph node metastasis, Fisher's exact test $\mathrm{F}=1.004, \mathrm{P}=0.318$; Table IV).

The rate of VM in the poor histological grade group was $96.6 \%$, which was significantly higher than that in the well-differentiated histological grade group $\left(61.1 \% ; \chi^{2}=9.202\right.$, $\mathrm{P}=0.002$ ). The $\mathrm{VM}$-positive rate in patients with stage I-III gallbladder carcinoma was $68.4 \%$ and that in patients with stage IV/V was $94.1 \%$, with a significant difference (Fisher's exact test $\mathrm{F}=8.324, \mathrm{P}=0.007$; Table IV).

Correlation between VM and STAT3 in gallbladder carcinoma. In the present study, VM was present in 52 of the 60 cases of gallbladder carcinoma with positive STAT3 expression $(86.7 \%)$.
In addition, among the 12 patients with negative STAT3 expression in gallbladder carcinoma tissues, 6 exhibited VM, with a rate of $50.0 \%$. Spearman's rank correlation analysis indicated a close and positive correlation between STAT3 expression and $\mathrm{VM}$ in gallbladder carcinoma $(\mathrm{R}=0.345, \mathrm{P}<0.05$; Table $\mathrm{V})$.

STAT3 expression is associated with early postoperative recurrence of gallbladder carcinoma. The relationship between the expression level of STAT3 and time to postoperative recurrence (TTR) of gallbladder carcinoma was then analyzed. As staining for STAT3 was mild to moderate in chronic cholecystitis tissues and its expression was significantly increased in carcinoma tissues, the cut-off value for STAT3 was calculated using a receiver operating 
Table IV. Association between VM and clinicopathological factors in patients with gallbladder carcinoma.

\begin{tabular}{|c|c|c|c|c|c|c|}
\hline \multirow[b]{2}{*}{ Factor } & \multirow[b]{2}{*}{$\mathrm{n}$} & \multicolumn{2}{|c|}{ VM } & \multirow{2}{*}{$\begin{array}{l}\text { Positive } \\
\text { rate }(\%)\end{array}$} & \multirow{2}{*}{$\begin{array}{c}\chi^{2} / \text { Fisher's exact } \\
\text { test value }\end{array}$} & \multirow[b]{2}{*}{ P-value } \\
\hline & & Positive & Negative & & & \\
\hline Sex & & & & & 0.010 & 0.920 \\
\hline Male & 30 & 24 & 6 & 80.0 & & \\
\hline Female & 42 & 34 & 8 & 81.0 & & \\
\hline Age (years) & & & & & 0.217 & 0.641 \\
\hline$<60$ & 32 & 25 & 7 & 78.1 & & \\
\hline$\geq 60$ & 40 & 33 & 7 & 82.5 & & \\
\hline Histological grade & & & & & 9.202 & 0.002 \\
\hline Well & 18 & 11 & 7 & 61.1 & & \\
\hline Moderate & 25 & 19 & 6 & 76.0 & & \\
\hline Poor & 29 & 28 & 1 & 96.6 & & \\
\hline Lymph node metastasis & & & & & 1.004 & 0.318 \\
\hline $\mathrm{N} 1+2$ & 54 & 45 & 9 & 83.3 & & \\
\hline N0 & 18 & 13 & 5 & 72.2 & & \\
\hline Clinical stage & & & & & 8.324 & 0.007 \\
\hline I -III & 38 & 26 & 12 & 68.4 & & \\
\hline IV-V & 34 & 32 & 2 & 94.1 & & \\
\hline
\end{tabular}

P-values for age, sex and histological grade were calculated by Pearson's $\chi^{2}$ test and those for lymph node metastasis and clinical stage were calculated by Fisher's exact test. VM, vasculogenic mimicry.

Table V. Correlation between VM and STAT3 in gallbladder carcinoma.

\begin{tabular}{lccccc}
\hline & & \multicolumn{3}{c}{ VM } & \\
\cline { 3 - 5 } STAT3 expression & Total & Positive & Negative & Positive rate (\%) & r \\
\hline Positive & 60 & 52 & 8 & 86.7 & 0.345 \\
Negative & 12 & 6 & 6 & 50.0 & 0.003 \\
Total & 72 & 58 & 14 & 80.6 & \\
\hline
\end{tabular}

VM, vasculogenic mimicry; STAT3, signal transducer and activator of transcription 3.

characteristic curve to evaluate the expression level of STAT3 in liver cancer tissues. The semi-quantitative cut-off value of the combined factor score was generated by combining two immunohistochemical diagnostic methods for STAT3, which were the coloration intensity diagnostic score and the number of stained cells diagnostic score. STAT3 diagnostic score $>3.49$ was regarded as high expression. Among them, 12 cases had low expression and 39 cases had high expression. The Youden index, which is a method to evaluate the authenticity of screening tests, was used (22). The Youden index of the semi-quantitative integration method for coloring intensity diagnosis $=0.971$, the Youden index of the number of stained cells diagnostic $=0.774$ and Joint Youden index $=1$ (Fig. 4A). Among the 51 patients with gallbladder carcinoma who completed a 3-year follow-up, the mean TTR of patients in the high-STAT3 group was 17.35 months and that in the low-STAT3 group was 35.65 months, with a significant difference $(\mathrm{P}<0.001$; Fig. 4B). A total of 8 patients were followed up for 36 months without postoperative recurrence, but the statistical data were analyzed according to the time of postoperative recurrence for 36 months. Thus, increased expression of STAT3 in gallbladder carcinoma tissue indicated early postoperative recurrence of gallbladder carcinoma.

$V M$ is associated with early postoperative recurrence of gallbladder carcinoma. The VM structure was detected in $47(92.15 \%, 47 / 51)$ of 51 cases of gallbladder carcinoma with 3 years of follow-up, with an average TTR of 22.38 months. The average TTR of the four cases $(7.84 \%, 4 / 51)$ with no VM structure was 36 months, because 4 patients with no VM structure were followed up for 36 months and exhibited no recurrence, but the statistical data were analyzed according to the time of postoperative recurrence for 36 months (Fig. 5). VM-positive gallbladder carcinoma cases were more likely to have early postoperative recurrence and there was a significant difference in TTR between the two groups $(\mathrm{P}<0.05)$. 

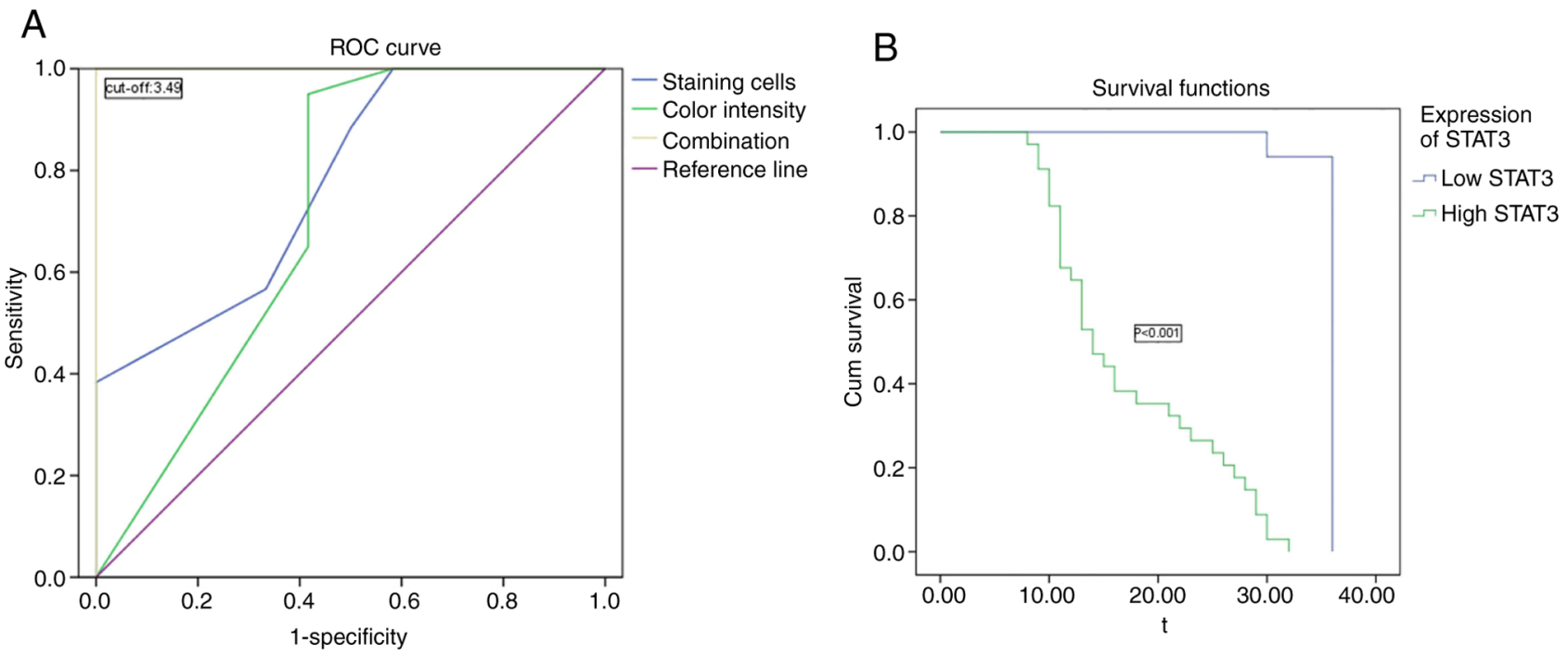

Figure 4. (A) ROC curve analysis was employed to estimate the cut-off points of the gallbladder carcinoma scores for STAT3 in tumor tissues. (B) High STAT3 expression indicates earlier postoperative recurrence. Kaplan-Meier curves were drawn and the log-rank test was utilized to compare the time to postoperative recurrence between the subgroups (high STAT3 vs. low STAT3). STAT3, signal transducer and activator of transcription 3; ROC, receiver operating characteristic; Cum, cumulative.

\section{Discussion}

To date, surgical resection remains the major treatment for gallbladder carcinoma, supplemented by adjuvant treatments such as chemotherapy or radiotherapy. The surgical mode for gallbladder carcinoma treatment should be determined according to its clinical stage. For patients with lesions limited to the gallbladder mucosa, conventional cholecystectomy may achieve an optimum therapeutic effect (23). Gemcitabine and platinum-based chemotherapy combined with molecular targeted therapy has become a promising avenue of research for treating gallbladder carcinoma. Targeted therapy utilizes a specific signaling molecule overexpressed on the surfaces of tumor cells as a target, and through the selection of specific blockers to interfere with the signal transduction pathway regulated by this molecule, it may inhibit the growth and invasion of tumors. Currently, the major targets for biliary system malignancies include epidermal growth factor receptor, vascular endothelial factor (VEGF) and mitogen-activated protein kinase $(24,25)$. Identifying targets for gallbladder carcinoma treatment is the basis of targeted therapy.

STAT3 was purified as an acute-phase response factor in interleukin-6 (IL-6) signaling and is a member of the tyrosine phosphorylation-activated family (26). IL-6 promotes tumor development by various mechanisms, for example, regulating the cell cycle, local inflammation, angiogenesis and tumor stem cell self-renewal (27). Numerous studies have indicated that STAT3 has a crucial role in the proliferation, invasion, metastasis, angiogenesis and immune escape of numerous types of tumor cell (28). Zhang et al (29) reported that Tanshinone IIA (Tan IIA) exerted potent anticancer activity in gastric cancer cells, mediated by downregulation of STAT3 activation. Overexpression of STAT3 significantly ameliorated the Tan IIA-induced suppression of cell growth and apoptosis. In addition, STAT3 is able to promote the proliferation of biliary tumors. It was reported that upregulation of STAT3 expression significantly enhanced the proliferation activity of

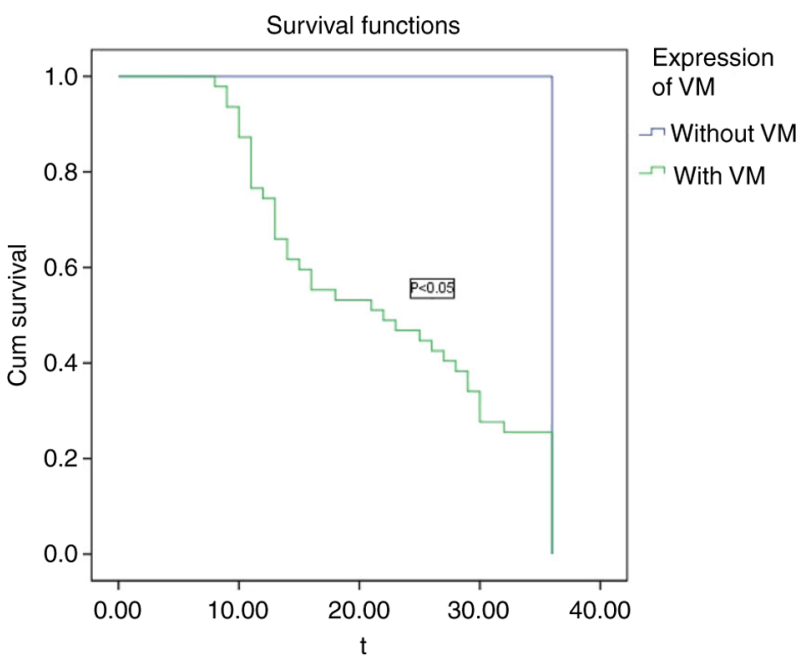

Figure 5. Presence of VM indicates earlier postoperative recurrence. KaplanMeier curves were drawn and the log-rank test was utilized to compare the time to postoperative recurrence between the subgroups (with VM vs. without VM). VM, vasculogenic mimicry; Cum, cumulative.

RBE and 9810 cell lines of bile duct cancer, whereas inhibition of STAT3 expression reduced the proliferation activity of the two cell lines (30). These results indicated that activation of STAT3 may promote the growth of tumor cells. Thus, inhibiting the expression and activation of STAT3 may be an effective approach for preventing the proliferation of tumor cells.

Invasion and metastasis are the major characteristics that distinguish malignant tumors from benign lesions (31). Tumor invasion and metastasis are the result of multiple factors. Neovascularization is a key step in this process and is regulated by various cytokines and growth factors, among which VEGF is the most important (32). VEGF produced by tumor cells is able to bind to tyrosine kinase receptors and promote the formation of new blood vessels, while STAT3 is a direct 
factor in activating VEGF expression (33). As early as in 2002, Niu et al (5) reported that activated STAT3 upregulated the expression of VEGF and promoted the formation of blood vessels in melanoma. STAT3 also regulated the migration of lymphatic endothelial cells and formation of lymphatic vascular lumen by upregulating the expression of VEGF and assisted tumor cells in lymphatic metastasis (34). Accumulating evidence has indicated that targeted inhibition of STAT3 is able to enhance the anticancer immune response and preserve the suppressed immune microenvironment in tumors, making it a promising target in cancer immunotherapy (35).

STAT3 is highly expressed in various cancer types, including endometrial cancer (36), glioma of the brain (37), breast cancer (38) and lung cancer (39). However, studies focusing on the relationship between STAT3 and gallbladder carcinoma are currently limited. Enyu et al (40) detected the expression of STAT3 in gallbladder carcinoma tissues and paraneoplastic tissues and determined that STAT3 expression was abnormally high in gallbladder carcinoma tissues. In the present study, the expression of STAT3 in gallbladder carcinoma tissues was also significantly higher than that in chronic cholecystitis tissues, with a positive expression rate of $83.3 \%$. These results are consistent with those of the study by Enyu et al (40), who suggested that STAT3 is related to the occurrence and development of gallbladder carcinoma. Furthermore, in the present study, the positive expression rate of STAT3 in chronic cholecystitis was $10.0 \%$, while the small sample size of the control group is a limitation of the present study. In addition, the STAT3 expression rate in stage I-III gallbladder carcinoma was $73.7 \%$ and that in stage IV/V, it was $94.1 \%$. In highly, moderately and poorly differentiated gallbladder carcinoma, the expression rates of STAT3 were $66.7,80.0$ and $96.6 \%$, respectively; the expression rate of STAT3 increased with decreased differentiation and these differences were significant $(\mathrm{P}<0.05)$. High expression of STAT3 was correlated with the clinical stage and histological grade of gallbladder carcinoma. However, no significant differences in STAT3 expression in gallbladder carcinoma tissues were obtained between different sexes, nor between different age and lymph node metastasis groups ( $\mathrm{P}>0.05)$. In the present study, 51 patients with gallbladder carcinoma who completed a 3-year follow-up had an average TTR of 17.353 months for high STAT3 expression and 35.647 months for low STAT3 expression. The results indicated that increased expression of STAT3 in gallbladder carcinoma tissues was positively associated with early postoperative recurrence of the malignancy. Due to the small sample size and follow-up time in the present study, the study results may be affected. In the future, the sample size should be increased, and the follow-up time should be extended to obtain more accurate research results.

Angiogenesis has been widely recognized as an important factor in the growth of solid tumors and angiogenesis has been widely studied in tumor therapy. VM, as a tumor vascular model that differs from angiogenesis, has been detected in melanoma, inflammatory breast cancer, liver cancer and other malignant tumor types with high invasive characteristics (41-43). VM has a completely different blood supply mode from classical tumor angiogenesis. Tumor cells imitate the blood vessels in the body to form a 'pipe-like' structure. There is no endothelial cell covering in the tube, but blood is able to flow through the 'pipe'. Since VM morphology in chromatoma was first described, numerous basic studies have focused on the formation mechanism of VM (44).

Hypoxia is one of the key conditions inducing VM formation and the hypoxia-inducible factor is highly localized in the VM region of tumors (45). Hypoxia is able to directly regulate the expression of genes such as those encoding VEGF-A, VEGF receptor 1, EPH receptor A2, Twist, Nodal, osteopontin and cyclooxygenase-2. It may also directly promote VM formation (46) and tumor metastasis (47). The present study indicated that the VM rate in the low-differentiation group was $96.6 \%$, which was significantly higher than that in the high-differentiation group (61.1\%). Therefore, the incidence of VM in highly malignant gallbladder carcinoma is higher than that in low malignant gallbladder carcinoma, which may be related to differences in gene expression and the cell phenotype. In addition, the VM-positive rate in stage I-III gallbladder carcinoma was $68.4 \%$, while it was $94.1 \%$ in stage IV/V, with a significant difference $(\mathrm{P}<0.05)$. This indicates that $\mathrm{VM}$ was correlated with the clinical stage of gallbladder carcinoma, with later clinical stages exhibiting higher VM positivity.

In clinical practice, patients with early gallbladder carcinoma may have liver metastasis and lymph node metastasis, and early recurrence after surgery may be associated with poor prognosis. This is consistent with the present results, according to which the VM structure was detected in $47(92.15 \%)$ of 51 cases of gallbladder carcinoma when followed up for 36 months after surgery and the mean TTR was 22.38 months. A total of 4 patients with no VM structure were followed up for 36 months and had no immediate recurrence after surgery, which may limit the results of the present study. Thus, patients with VM-positive gallbladder carcinoma were more likely to have early postoperative recurrence. These results suggested that VM formation has an important role in the progression of gallbladder carcinoma. In addition, there were no significant differences in VM expression in gallbladder carcinoma tissues between patients with different sexes, nor between different age and lymph node metastasis groups $(\mathrm{P}>0.05)$. According to the currently known results of clinical applications, neutralizing monoclonal antibodies and small-molecule drugs targeting angiogenesis-promoting factors such as VEGF, platelet-derived growth factor and fibroblast growth factor are not able to significantly reduce the postoperative recurrence rate and prolong the survival time (48). By contrast, inhibition of EDV leads to increased hypoxia in the tumor, aggravating the phenotypic deterioration of tumor cells and promoting the formation of VM (49-51). Therefore, studies to evaluate therapeutic strategies for inhibiting VM in gallbladder carcinoma and identify drugs targeting key molecules in the process of VM formation are required.

In the present study, the expression of STAT3 and VM in gallbladder carcinoma tissues was significantly increased and positively correlated. In gallbladder carcinoma tissues, a lower degree of differentiation, higher malignancy degree and higher clinical stage of malignancy were associated with higher expression rates of STAT3 and VM. This may indicate that STAT3 directly or indirectly promotes the structure formation of VM, thus having an important role in the process of tumor occurrence, development and metastasis. 
It can be concluded from the present study that VM was associated with poor prognosis of gallbladder carcinoma. As activation of STAT3 increased the expression of VEGF to promote the formation of tumor blood vessels, increases in the expression of STAT3 may promote the formation of gallbladder carcinoma. Future studies by our group will examine the roles of STAT3 in gallbladder carcinoma and VM, as well as their possible mechanisms of action. By regulating the expression levels of STAT3 in gallbladder carcinoma cells, changes in the expression of STAT3 in gallbladder carcinoma and the influence of VM may be determined. STAT3, as a regulatory target, may inhibit the proliferation and invasion of tumor cells and block the development of VM and EDV, thereby representing a suitable target for antitumor angiogenesis therapy.

\section{Acknowledgements}

The authors thank Dr Qian Haixin from the Department of General Surgery (The First Affiliated Hospital of Soochow University, Suzhou, China) for guidance with the study design and technical support.

\section{Funding}

This work was supported by a grant from The Fifth Affiliated Hospital of the Medical School of Nantong University (Taizhou, China; grant no. ZL201931).

\section{Availability of data and materials}

The datasets used and/or analyzed during the present study are available from the corresponding author on reasonable request.

\section{Authors' contributions}

HZ and HQ designed experiments and wrote the manuscript. $\mathrm{HZ}$ and YY performed experiments and provided technical support. YY and HQ confirmed the authenticity of the raw data. All authors read and approved the final manuscript.

\section{Ethics approval and consent to participate}

This study was reviewed and approved by the ethics committee of The Fifth Affiliated Hospital of the Medical School of Nantong University (Taizhou, China). All patients participating in the present study or their family members signed an informed consent form to participate in the clinical study.

\section{Patient consent for publication}

Not applicable.

\section{Competing interests}

The authors declare that they have no competing interests.

\section{References}

1. Hundal R and Shaffer EA: Galltesticles Cancer: Epidemiology and outcome. Clin Epidemiol 6: 99-109, 2014.
2. Chen W, Zheng R, Baade PD, Zhang S, Zeng H, Bray F, Jemal A, Yu XQ and He J: Cancer statistics in China, 2015. CA Cancer J Clin 66: 115-132, 2016.

3. Liu S, Wang X and Liu Y: Advances in comprehensive treatment of gallbladder cancer. Surgery Concepts \& Practice 21: 365-368, 2016.

4. Folkman J: Tumor angiogenesis: Therapeutic implications. N Engl J Med 285: 1182-1186, 1971.

5. Niu G, Wright KL, Huang M, Song L, Haura E, Turkson J, Zhang S, Wang T, Sinibaldi D, Coppola D, et al: Constitutive Stat3 activity up-regulates VEGF expression and tumor angiogenesis. Oncogene 21: 2000-2008, 2002.

6. Lee SE, Jang JY, Lim CS, Kang MJ and Kim SW: Systematic review on the surgical treatment for T1 gallbladder cancer. World J Gastroenterol 17: 174-180, 2011.

7. Isambert M, Leux C, Métairie S and Paineau J: Incidentallydiscovered gallbladder cancer: When, why and which reoperation. J Visc Surg 148: e77-e84, 2011.

8. Cavallaro A, Piccolo G, Panebianco V, Lo Menzo E, Berretta M, Zanghì A, Di Vita $\mathrm{M}$ and Cappellani A: Incidental gallbladder cancer during laparoscopic cholecystectomy: Managing an unexpected finding. World J Gastroenterol 18: 4019-4027, 2012.

9. Horiguchi A, Miyakawa S, Ishihara S, Miyazaki M, Ohtsuka M, Shimizu H, Sano K, Miura F, Ohta T, Kayahara M, et al: Gallbladder bed resection or hepatectomy of segments $4 \mathrm{a}$ and 5 for pT2 gallbladder carcinoma: Analysis of Japanese registration cases by the study group for biliary surgery of the Japanese Society of Hepato-Biliary-Pancreatic Surgery. J Hepatobiliary Pancreat Sci 20: 518-524, 2013

10. Fathi N, Rashidi G, Khodadadi A, Shahi S and Sharifi S: STAT3 and apoptosis challenges in cancer. Int J Biol Macromol 117: 993-1001, 2018.

11. Kitamura H, Ohno Y, Toyoshima Y, Ohtake J, Homma S, Kawamura H, Takahashi N and Taketomi A: Interleukin-6/STAT3 signaling as a promising target to improve the efficacy of cancer immunotherapy. Cancer Sci 108: 1947-1952, 2017.

12. Nagakura S, Shirai Y, Yokoyama N and Hatakeyama K: Clinical significance of lymph node micrometastasis in gallbladder carcinoma. Surgery 129: 704-713, 2001

13. Wang H, Zhan M, Liu Q and Wang J: Glycochenodeoxycholate promotes the metastasis of gallbladder cancer cells by inducing epithelial to mesenchymal transition via activation of SOCS3/JAK2/STAT3 signaling pathway. J Cell Physiol 235: 1615-1623, 2020.

14. Fernandez-Cortes M, Delgado-Bellido D and Oliver FJ: Vasculogenic mimicry: Become an endothelial cell 'but not so much'. Front Oncol 9: 803-809, 2019.

15. Kim SH, Lee HS, Kang BJ, Song BJ, Kim HB, Lee H, Jin MS and Lee A: Dynamic contrast-enhanced MRI perfusion parameters as imaging biomarkers of angiogenesis. PLoS One 11: E0168632, 2016.

16. Dutta S, Going JJ, Crumley AB, Mohammed Z, Orange C, Edwards J, Fullarton GM, Horgan PG and McMillan DC: The relationship between tumour necrosis, tumour proliferation, local and systemic inflammation, microvessel density and survival in patients undergoing potentially curative resection of oesophageal adenocarcinoma. Brit J Cancer 106: 702-710, 2012.

17. Lazcano-Ponce EC, Miquel JF, Muñoz N, Herrero R, Ferrecio C, Wistuba II, Alonso de Ruiz P, Aristi Urista G and Nervi F: Epidemiology and molecular pathology of gallbladder cancer. CA Cancer J Clin 51: 349-364, 2001

18. Lesina M, Kurkowski MU, Ludes K, Rose-John S, Treiber M, Klöppel G, Yoshimura A, Reindl W, Sipos B, Akira S, et al: Stat3/Socs 3 activation by IL- 6 transsignaling promotes progression of pancreatic intraepithelial neoplasia and development of pancreatic cancer. Cancer Cell 19: 456-69, 2011.

19. EdgeS,ByrdDR,ComptonCC,Fritz AG,Greene Fand Trotti A(eds): AJCC Cancer Staging Manual. 7th edition, Springer, New York, 2010. https://www.springer.com/us/book/9780387884424.

20. Maniotis AJ, Folberg R, Hess A, Seftor EA, Gardner LM, Pe'er J, Trent JM, Meltzer PS and Hendrix MJ: Vascular channel formation by human melanoma cells in vivo and in vitro: Vasculogenic mimicry. Am J Pathol 155: 739-752, 1999.

21. Okayasu I and Hara A: Cyclooxygenase-2 and inducible nitric oxide synthase expression in human astrocytic gliomas: Correlation with angiogenesis and prognostic significance. Acta Neuropathol 108: 43-48, 2004.

22. Li C, Chen J and Qin G: Partial Youden index and its inferences. J Biopharm Stat 29: 385-399, 2019. 
23. Lee SE, Jang JY, Lim CS, Kang MJ and Kim SW: Systematic review on the surgical treatment for $\mathrm{T} 1$ gallbladder cancer. World J Gastroenterol 17: 174-180, 2011.

24. Fratto ME, Santini D, Vincenzi B, Silvestris N, Azzariti A, Tommasi S, Zoccoli A, Galluzzo S, Maiello E, Colucci G and Tonini G: Targeting EGFR in bilio-pancreatic and liver carcinoma. Front Biosci (Schol Ed) 3: 16-22, 2011.

25. Chen Y, Jiang L, She F, Tang N, Wang X, Li X, Han S and Zhu J: Vascular endothelial growth factor-C, promotes the growth and invasion of gallbladder cancer via an autocrine mechanism. Mol Cell Biochem 345: 77-89, 2010.

26. Lirdprapamongkol K, Sakurai H, Abdelhamed S, Yokoyama S, Athikomkulchai S, Viriyaroj A, Awale S, Ruchirawat S, Svasti J and Saiki I: Chrysin overcomes TRAIL resistance of cancer cells through Mcl-1 downregulation by inhibiting STAT3 phosphorylation. Int J Oncol 43: 329-337, 2013.

27. Wu YS, Chung I, Wong WF, Masamune A, Sim MS and Looi CY: Paracrine IL-6 signaling mediates the effects of pancreatic stellate cells on epithelial-mesenchymal transition via Stat3/Nrf2 pathway in pancreatic cancer cells. Biochim Biophys Acta Gen Subj 1861: 296-306, 2017.

28. O'Sullivan T, Saddawi-Konefka R, Vermi W, Koebel CM, Arthur C, White JM, Uppaluri R, Andrews DM, Ngiow SF, Teng MW, et al: Cancer immunoediting by the innate immune system in the absence of adaptive immunity. J Exp Med 209: 1869-1882, 2012.

29. Zhang Y, Guo S, Fang J, Peng B, Zhang Y and Cao T: Tanshinone IIA inhibits cell proliferation and tumor growth by downregulating STAT3 in human gastric cancer. Exp Ther Med 16: 2931-2937, 2018

30. Ke F, Wang Z, Song X, Ma Q, Hu Y, Jiang L, Zhang Y, Liu Y, Zhang $\mathrm{Y}$ and Gong W: Cryptotanshinone induces cell cycle arrest and apoptosis through the JAK2/STAT3 and PI3K/Akt/NFKB pathways in cholangiocarcinoma cells. Drug Des Devel Ther 11: 1753-1766, 2017.

31. Ruiz P and Günthert U: The cellular basis of metastasis. World J Urol 14: 141-150, 1996.

32. Sohn EJ, Jung DB, Lee H, Han I, Lee J, Lee H and Kim SH: CNOT2 promotes proliferation and angiogenesis via VEGF signaling in MDA-MB-231 breast cancer cells. Cancer Lett 412 88-98, 2018.

33. Chen Z and Han ZC: STAT3: A critical transcription activator in angiogenesis. Med Res Rev 28: 185-200, 2008.

34. Okazaki H, Tokumaru S, Hanakawa Y, Shiraishi K, Shirakata Y, Dai X, Yang L, Tohyama M, Hashimoto K and Sayama K: Nuclear translocation of phosphorylated STAT3 regulates VEGF-A-induced lymphatic endothelial cell migration and tube formation. Biochem Biophys Res Commun 412: 441-445, 2011.

35. Wang Y, Shen Y, Wang S, Shen Q and Zhou X: The role of STAT3 in leading the crosstalk between human cancers and the immune system. Cancer Lett 415: 117-128, 2018.

36. Bao W, Wang HH, Tian FJ, He XY, Qiu MT, Wang JY, Zhang HJ, Wang LH and Wan XP: A TrkB-STAT3-miR-204-5p regulatory circuitry controls proliferation and invasion of endometrial carcinoma cells. Mol Cancer 12: 155, 2013.

37. Kruczyk M, Przanowski P, Dabrowski M, Swiatek-Machado K, Mieczkowski J, Wallerman O, Ronowicz A, Piotrowski A, Wadelius C, Kaminska B and Komorowski J: Integration of genome-wide of Stat3 binding and epigenetic modification mapping with transcriptome reveals novel Stat 3 target genes in glioma cells. Biochim Biophys Acta 1839: 1341-1350, 2014.
38. Cheng GZ, Zhang WZ, Sun M, Wang Q, Coppola D, Mansour M, $\mathrm{Xu}$ LM, Costanzo C, Cheng JQ and Wang LH: Twist is transcriptionally induced by activation of STAT3 and mediates STAT3 oncogenic function. J Biol Chem 283: 14665-14673, 2008.

39. Cao C, Zhao G, Yu W, Xie X, Wang W, Yang R, Lv X and Liu D: Activation of STAT3 stimulates AHSP expression in K562 cells. Sci China Life Sci 57: 488-494, 2014.

40. Enyu L, Na W, Chuanzong Z, Ben W, Xiaojuan W, Yan W, Zequn L, Jianguo H, Jiayong W, Benjia L, et al: The clinical significance and underlying correlation of pStat-3 and integrin $\alpha v \beta 6$ expression in gallbladder cancer. Oncotarget 8: 19467-19477, 2017

41. Sun B, Zhang D, Zhao N and Zhao X: Epithelial-to-endothelial transition and cancer stem cells: Two cornerstones of vasculogenic mimicry in malignant tumors. Oncotarget 8: 30502-30510, 2017.

42. Vartanian A, Karshieva S, Dombrovsky V and Belyavsky A: Melanoma educates mesenchymal stromal cells towards vasculogenic mimicry. Oncol Lett 11: 4264-4268, 2016.

43. Pereira JA, Bilhim T, Rio Tinto H, Fernandes L, Martins Pisco J and Goyri-O'Neill J: Radiologic anatomy of arteriogenic erectile dysfunction: A systematized approach. Acta Med Port 26: 219-225, 2013.

44. Bissell MJ: Tumor plasticity allows vasculogenic mimicry, a novel form of angiogenic switch. A rose by anyother name? Am J Pathol 155: 675-679, 1999.

45. Wang HF, Wang SS, Zheng M, Dai LL, Wang K, Gao XL, Cao MX, Yu XH, Pang X, Zhang M, et al: Hypoxia promotes vasculogenic mimicry formation by vascular endothelial growth factor A mediating epithelial-mesenchymal transition in salivary adenoid cystic carcinoma. Cell Prolif 52: e12600, 2019.

46. Li S, Meng W, Guan Z, Guo Y and Han X: The hypoxia-related signaling pathways of vasculogenic mimicryin tumor treatment Biomed Pharmacother 80: 127-135, 2016.

47. Liu K, Sun B, Zhao X, Wang X, Li Y, Qiu Z, Liu T, Gu Q, Dong X, Zhang Y, et al: Hypoxia promotes vasculogenic mimicry formation by the Twist1-Bmil connection in hepatocellular carcinoma. Int J Mol Med 36: 783-791, 2015.

48. Sun H, Zhang D, Yao Z, Lin X, Liu J, Gu Q, Dong X, Liu F, Wang Y, Yao N, et al: Anti-angiogenic treatment promotes triple-negative breast cancer invasion via vasculogenic mimicry. Cancer Biol Ther 18: 205-213, 2017.

49. Hendrix MJ, Seflor EA, Seflor RE, Chao JT, Chien DS and Chu YW: Tumor cell vascular mimicry: Novel targeting opportunity in melanoma. Pharmacol Ther 159: 83-92, 2016.

50. Huang B, Xiao E and Huang M: MEK/ERK pathway is positively involved in hypoxia-induced vasculogenic mimicry formation in hepatocellular carcinoma which is regulated negatively by protein kinase A. Med Oncol 32: 408, 2015.

51. Vacca A, Ria R, Reale A and Ribatti D: Angiogenesis in multiple myeloma. Chem Immunol Allergy 99: 180-196, 2014.

This work is licensed under a Creative Commons Attribution-NonCommercial-NoDerivatives 4.0 International (CC BY-NC-ND 4.0) License. 